\title{
A Theoretical Model of Overpotential at Interfaces in Polymer Electrolyte Fuel Cells
}

\author{
Kentaro Doi, Hiroki Hashizume, and Satoyuki Kawano
}

\begin{abstract}
Polymer electrolyte fuel cells (PEFCs) have recently attracted considerable attention as a promising clean energy source. In the present study, we develop a theoretical model for the overpotential in PEFCs. Protons with sufficient kinetic energy to overcome the potential barrier can pass through the interfaces. They then converge to a constant current, dissipating their kinetic energy. In particular, inelastic collisions of protons at electrode-electrolyte interfaces are considered to be the dominant cause of overvoltage. This model based on the assumptions above differs from conventional continuum models. The effects of potential barriers at interfaces, proton concentrations, temperature, and mass on the voltage loss are clarified by a parameter study. To verify this model, we prepare two different membrane electrode assemblies (MEAs) that have different interface conditions and measure their current-voltage characteristics. As a result, substantial voltage losses from the MEA with the poorer quality interfaces are observed. Theoretical evaluations of the overvoltage are in good agreement with the experimental results. The present results imply that it is essential to control interface conditions to achieve high proton conduction.
\end{abstract}

Index Terms-Polymer electrolyte fuel cell (PEFC), overpotential, membrane electrode assembly (MEA), interfaces.

\section{INTRODUCTION}

Polymer electrolyte fuel cells (PEFCs) are especially attractive because they have relatively low operating temperature, are portable, and are safe to use. Therefore, such an energy conversion system is expected to be used as efficient power sources in the near future. Many experimental and theoretical studies have been conducted on PEFCs with the aim of developing them for practical applications [1], [2]. On the other hand, several problems have remained to be solved, including overpotential at high-load operation, humidification, and the need to achieve efficient activation of $\mathrm{H}_{2}$ and $\mathrm{O}_{2}$.

To increase the energy conversion efficiency, it is important to determine the overpotential generation mechanism and to develop effective solutions for suppressing the energy losses. In previous studies, proton conduction mechanisms in PEFCs were modeled using continuum models based on fluid mechanics, mass transport, and electrodynamics [3], [4]. These theoretical models can explain the current-voltage characteristics of PEFCs. These models have been emphasized by some groups [5]-[7].

Manuscript received September 1, 2014; revised November 7, 2014.

K. Doi and S. Kawano are with the Department of Mechanical Science and Bioengineering, Osaka University, 1-3 Machikaneyama, Toyonaka, Osaka 560-8531, Japan (e-mail: doi@me.es.osaka-u.ac.jp, kawano@me.es.osaka-u.ac.jp).

H. Hashizume is with the Graduate School of Engineering Science, Osaka University, 1-3 Machikaneyama, Toyonaka, Osaka 560-8531, Japan.
However, their complexity makes it difficult to propose ways to overcome the problems in PEFCs. In addition, those models disregard atomistic-scale phenomena. On the other hand, recent reports [8]-[11] have emphasized the importance of understanding proton conduction mechanisms by focusing on the behavior of discrete charged particles. Atomistic-scale investigations that exploit recent advances in nanoscale fabrication techniques are expected to clarify the mechanisms of various phenomena in PEFCs, resulting in breakthroughs.

In the present study, a theoretical model is developed to explain the nature of overpotentials in PEFCs from a viewpoint of atomistic-scale phenomena. The carrier conduction mechanism is treated based on proton kinetics. Inelastic collisions of protons at electrode-electrolyte interfaces are considered to be the origin of overpotential. The overpotentials depend on the transports of carriers distributed between the two layers. As a first step, our model assumes a Maxwell distribution of protons in local equilibrium. Nonequilibrium relaxation processes are then taken into account to represent the energy dissipation caused by inelastic collisions at the interfaces.

This theoretical model successfully explains the results from experimental measurements. In particular, different interface conditions in artificial preparations can be represented and the voltage drop is attributed to inelastic collisions of protons at interfaces. The barrier heights and proton concentrations in each layer are found to critically affect the overpotential. Consequently, the energy dissipation at electrode-electrolyte interfaces is the main source of overpotential in PEFCs; thus, fabricating seamless interfaces are suggested to be the most important way to improve the performance of PEFCs.

\section{TheORETICAL Model AND Method OF SOlution}

In conventional models, overpotentials in PEFCs are usually explained in terms of continuum models in which proton diffusion, mass transport, and electric conduction are taken into account [3], [4]. However, in recent decades, microscale analyses have been conducted in which proton behavior is modeled by atomic interactions. First-principles electronic-structure calculations have been performed to investigate proton transfer through electrolyte membranes [11]. Herein, overpotentials in PEFCs are evaluated using a theoretical model in which inelastic collisions of protons at electrode-electrolyte interfaces give rise to energy losses. We suppose an idea to treat the overpotential due to proton transport in non-equilibrium current condition. Fig. 1 shows a schematic illustration of this model. The proton number densities in the anode, electrolyte, and cathode layers are 
represented by $n_{\mathrm{A}}, n_{\mathrm{E}}$, and $n_{\mathrm{C}}$, respectively. The number of protons that collide with the anode-electrolyte interface from the anode side per unit area per unit time, that is number flux, is represented by $f_{\mathrm{A}}^{+}$and that from the electrolyte side is represented by $f_{\mathrm{E}}^{-}$. In the same manner, the number of protons that collide with the cathode-electrolyte interface from the cathode side is represented by $f_{\mathrm{C}}{ }^{-}$and that from the electrolyte side is represented by $f_{\mathrm{E}}^{+}$. Protons that have sufficient kinetic energy to overcome the potential barriers at the interfaces pass through the anode-electrolyte or cathodeelectrolyte interface. The inelastic collision processes can be expressed as follows:

$$
\begin{gathered}
\frac{\mathrm{d} f_{\mathrm{A}}^{+}}{\mathrm{d} t}=-k_{1 \mathrm{f}} f_{\mathrm{A}}^{+}+k_{1 \mathrm{r}} f_{\mathrm{E}}^{-}, \\
\frac{\mathrm{d} f_{\mathrm{E}}^{-}}{\mathrm{d} t}=k_{1 \mathrm{f}} f_{\mathrm{A}}^{+}-k_{1 \mathrm{r}} f_{\mathrm{E}}^{-}, \\
\frac{\mathrm{d} f_{\mathrm{E}}^{+}}{\mathrm{d} t}=-k_{2 \mathrm{f}} f_{\mathrm{E}}^{+}+k_{2 \mathrm{r}} f_{\mathrm{C}}^{-}, \\
\frac{\mathrm{d} f_{\mathrm{C}}^{-}}{\mathrm{d} t}=k_{2 \mathrm{f}} f_{\mathrm{E}}^{+}-k_{2 \mathrm{r}} f_{\mathrm{C}}^{-},
\end{gathered}
$$

and then

$$
f_{\mathrm{E}}=f_{\mathrm{E}}^{+}+f_{\mathrm{E}}^{-},
$$

where $k_{1 \mathrm{f}}, k_{1 \mathrm{r}}, k_{2 \mathrm{f}}$, and $k_{2 \mathrm{r}}$ are respectively the transition probabilities from the anode to the electrolyte, from the electrolyte to the anode, from the electrolyte to the cathode, and from the cathode to the electrolyte. The proton distributions are assumed to be in local equilibrium so that the transition probabilities $k_{i \mathrm{f}}$ and $k_{i \mathrm{r}}$ can be represented as

$$
\begin{array}{r}
k_{i \mathrm{f}}=\gamma_{i} \frac{\int_{v_{i \mathrm{f}}}^{\infty} v \exp \left[-\frac{\beta m}{2}\left(v-v_{\mu}\right)^{2}\right] d v}{\int_{0}^{\infty} v \exp \left[-\frac{\beta m}{2}\left(v-v_{\mu}\right)^{2}\right] d v}, \\
k_{i \mathrm{r}}=\gamma_{i} \frac{\int_{-\infty}^{-v_{i \mathrm{r}}} v \exp \left[-\frac{\beta m}{2}\left(v-v_{\mu}\right)^{2}\right] d v}{\int_{-\infty}^{0} v \exp \left[-\frac{\beta m}{2}\left(v-v_{\mu}\right)^{2}\right] d v},
\end{array}
$$

where $i=1$ or $2, v_{i f}, v_{i \mathrm{r}}>0, \gamma_{i}$ is the collision frequency at the interfaces, $m$ is the proton mass, $v_{\mu}$ and $v_{v}$ are respectively the mean proton velocities in the forward and backward direction in the anode $v_{\mathrm{A}}$, the cathode $v_{\mathrm{C}}$, or the electrolyte $v_{\mathrm{E}}$, and $\beta$ is $1 / k_{\mathrm{B}} T$ where $k_{\mathrm{B}}$ and $T$ are the Boltzmann constant and temperature, respectively. In the steady state, the proton flow is considered to be predominately one-dimensional. The velocity threshold $v_{\text {if }}$ is determined from the potential barrier heights at the electrode-electrolyte interfaces: $v_{i f}=\sqrt{2 \phi_{i} / m}$, where $\phi_{i}$ is the barrier height at the $i$ th interface, as shown in Fig. 1. In this study, the potential barriers are assumed to be the same for forward and backward flow of protons (i.e., $v_{i r}=$ $\left.v_{i f}\right)$. According to (6) and (7), the proton flux in each layer depends on the mean velocities, which are given by

$$
f_{\mathrm{A}}^{+}\left(v_{\mathrm{A}}\right)=n_{\mathrm{A}} \sqrt{\frac{\beta m}{2 \pi}} \int_{0}^{\infty} v \exp \left[-\frac{\beta m}{2}\left(v-v_{\mathrm{A}}\right)^{2}\right] \mathrm{d} v,
$$

$$
\begin{gathered}
f_{\mathrm{E}}^{-}\left(v_{\mathrm{E}}\right)=n_{\mathrm{E}} \sqrt{\frac{\beta m}{2 \pi}} \int_{-\infty}^{0} v \exp \left[-\frac{\beta m}{2}\left(v-v_{\mathrm{E}}\right)^{2}\right] \mathrm{d} v, \\
f_{\mathrm{E}}^{+}\left(v_{\mathrm{E}}\right)=n_{\mathrm{E}} \sqrt{\frac{\beta m}{2 \pi}} \int_{0}^{\infty} v \exp \left[-\frac{\beta m}{2}\left(v-v_{\mathrm{E}}\right)^{2}\right] \mathrm{d} v, \\
f_{\mathrm{C}}^{-}\left(v_{\mathrm{C}}\right)=n_{\mathrm{C}} \sqrt{\frac{\beta m}{2 \pi}} \int_{-\infty}^{0} v \exp \left[-\frac{\beta m}{2}\left(v-v_{\mathrm{C}}\right)^{2}\right] \mathrm{d} v,
\end{gathered}
$$

where $n_{\mathrm{A}}, n_{\mathrm{E}}$, and $n_{\mathrm{C}}$ are the number density of protons in the anode, electrolyte, and cathode, respectively. These distributions are used in (8)-(11) together with the assumption that protons in each layer frequently collide with each other and they form local Maxwell distributions, the centers of which are shifted by the mean velocities. In this study, we focus on momentum space because energy dissipation is dominated by momentum transfer through inelastic collisions at interfaces. Spatial distributions are assumed to be uniform very near the interfaces. However, the molecular structures in the interfaces should be determined in future studies and this information should be incorporated in a theoretical model to enable more realistic situations to be simulated. In experiments, the current-voltage characteristics are measured for a constant current density. Thus, the current density $j$ can be expressed by

$$
j=q n_{\mathrm{A}} v_{\mathrm{A}}=q n_{\mathrm{E}} v_{\mathrm{E}}=q n_{\mathrm{C}} v_{\mathrm{C}},
$$

where $q$ is the charge of proton. Based on (8)-(11), the left-hand sides of (1)-(4) can be expanded as follows:

$$
\begin{gathered}
\frac{\mathrm{d} f_{\mathrm{A}}^{+}}{\mathrm{d} t}=\frac{\partial f_{\mathrm{A}}^{+}}{\partial v_{\mathrm{A}}} \frac{\mathrm{d} v_{\mathrm{A}}}{\mathrm{d} t}=-\frac{j}{v_{\mathrm{A}}^{2} \sqrt{2 \pi \beta m}} \exp \left[-\frac{\beta m}{2} v_{\mathrm{A}}^{2}\right] \frac{\mathrm{d} v_{\mathrm{A}}}{\mathrm{d} t}, \\
\frac{\mathrm{d} f_{\mathrm{C}}^{ \pm}}{\mathrm{d} t}=\frac{\partial f_{\mathrm{C}}^{ \pm}}{\partial v_{\mathrm{C}}} \frac{\mathrm{d} v_{\mathrm{C}}}{\mathrm{d} t}=-\frac{j}{v_{\mathrm{C}}^{2} \sqrt{2 \pi \beta m}} \exp \left[-\frac{\beta m}{2} v_{\mathrm{C}}^{2}\right] \frac{\mathrm{d} v_{\mathrm{C}}}{\mathrm{d} t}, \\
\frac{\mathrm{d} f_{\mathrm{E}}}{\mathrm{d} t}=\frac{\partial}{\partial v_{\mathrm{E}}}\left(f_{\mathrm{E}}^{+}+f_{\mathrm{E}}^{-}\right) \frac{\mathrm{d} v_{\mathrm{E}}}{\mathrm{d} t} \\
=-\frac{2 j}{v_{\mathrm{E}}^{2} \sqrt{2 \pi \beta m}} \exp \left[-\frac{\beta m}{2} v_{\mathrm{E}}^{2}\right] \frac{\mathrm{d} v_{\mathrm{E}}}{\mathrm{d} t} .
\end{gathered}
$$

(a)
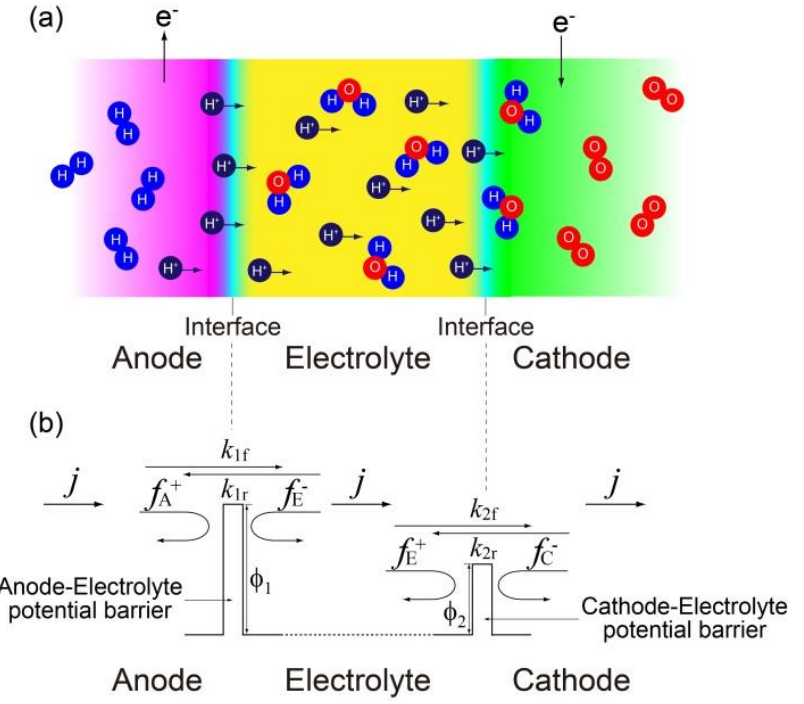

Fig. 1. Schematic illustration of proton transfer in MEA. The model assumes potential barriers and inelastic collisions at interfaces. 
Substituting (13)-(15) into (1)-(4), they result in the first-order reaction equations. When these equations are solved numerically, $v_{\mathrm{A}}, v_{\mathrm{C}}$, and $v_{\mathrm{E}}$ converge to the terminal velocities, enabling the distributions in (8)-(11) to be determined. Protons that overcome a potential barrier at an interface transfer to the adjacent layer and settle into local equilibrium. The energy dissipation at each interface can be evaluated from the relaxation process in proton transfer. For example, the energy dissipation of protons that pass through the anode to the electrolyte is defined as

$$
\begin{aligned}
W_{\mathrm{AE}}= & n_{\mathrm{A}} \sqrt{\frac{\beta m}{2 \pi}} \int_{v_{\mathrm{lf}}}^{\infty} \frac{1}{2} m v^{3} \exp \left[-\frac{\beta m}{2}\left(v-v_{\mathrm{A}}\right)^{2}\right] \mathrm{d} v \\
& -\frac{\beta m n_{\mathrm{A}}}{2 \pi} \int_{v_{\mathrm{lf}}}^{\infty} v_{1} \exp \left[-\frac{\beta m}{2}\left(v_{1}-v_{\mathrm{A}}\right)^{2}\right] \mathrm{d} v_{1} \\
& \times \int_{-\infty}^{\infty} \frac{1}{2} m v_{2}^{2} \exp \left[-\frac{\beta m}{2}\left(v_{2}-v_{\mathrm{E}}\right)^{2}\right] \mathrm{d} v_{2}
\end{aligned}
$$

and that caused by reverse flow is written as

$$
\begin{aligned}
W_{\mathrm{EA}} & =n_{\mathrm{E}} \sqrt{\frac{\beta m}{2 \pi}} \int_{-\infty}^{-v_{\mathrm{lf}}} \frac{1}{2} m|v|^{3} \exp \left[-\frac{\beta m}{2}\left(v-v_{\mathrm{E}}\right)^{2}\right] \mathrm{d} v \\
& -\frac{\beta m n_{\mathrm{E}}}{2 \pi} \int_{-\infty}^{-v_{\mathrm{lr}}}\left|v_{1}\right| \exp \left[-\frac{\beta m}{2}\left(v_{1}-v_{\mathrm{E}}\right)^{2}\right] \mathrm{d} v_{1} \\
& \times \int_{-\infty}^{\infty} \frac{1}{2} m v_{2}^{2} \exp \left[-\frac{\beta m}{2}\left(v_{2}-v_{\mathrm{A}}\right)^{2}\right] \mathrm{d} v_{2}
\end{aligned}
$$

The dissipation at the cathode-electrolyte interface is expressed in the same manner as (16) and (17). The overpotential from these interfaces is estimated by dividing the energy dissipation by the proton flux through the interfaces:

$$
V_{\mathrm{AE}} \equiv \frac{W_{\mathrm{AE}}}{q n_{\mathrm{I}} / \sqrt{\beta m}}=\frac{W_{\mathrm{AE}}}{\xi_{\mathrm{I}}}
$$

and

$$
V_{\mathrm{EA}} \equiv \frac{W_{\mathrm{EA}}}{q n_{\mathrm{I}} / \sqrt{\beta m}}=\frac{W_{\mathrm{EA}}}{\xi_{\mathrm{I}}},
$$

where $n_{\mathrm{I}}$ is the charge and the number density of protons at the anode-electrolyte interface, respectively. Protons at an interface are assumed to be affected by the interface conditions and temperature. However, discussion about the details of the interface is beyond the scope of this study and is left for future work because these parameters seem to depend on the electronic structures of the interface and should be determined using quantum chemical methods. In the present study, these quantities are replaced by the parameter $\xi_{\mathrm{I}}$. The overvoltage at the cathode-electrolyte interface is similarly given by

$$
V_{\mathrm{EC}} \equiv \frac{W_{\mathrm{EC}}}{q n_{\mathrm{I}^{\prime}} / \sqrt{\beta m}}=\frac{W_{\mathrm{EC}}}{\xi_{\mathrm{I}^{\prime}}}
$$

and

$$
V_{\mathrm{EC}} \equiv \frac{W_{\mathrm{EC}}}{q n_{\mathrm{I}^{\prime}} / \sqrt{\beta m}}=\frac{W_{\mathrm{EC}}}{\xi_{\mathrm{I}^{\prime}}},
$$

where $n_{\mathrm{I}}$ is the charge and the number density of protons at the cathode-electrolyte interface, respectively. The interface condition is represented by $\xi_{\mathrm{I}}$. Thus, in the present study, the overpotential from the interfaces is given by

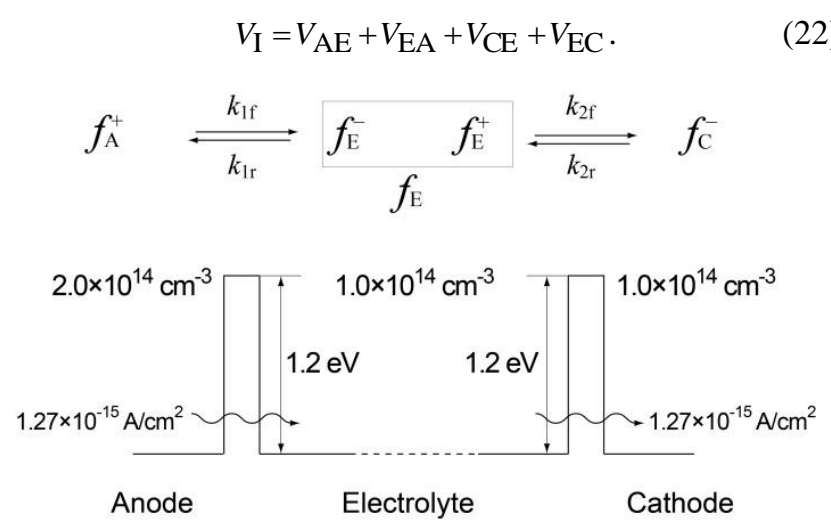

Fig. 2. Schematic diagram and parameters used to compute the reference state for results shown in Fig. 3. $n_{\mathrm{A}}, n_{\mathrm{E}}$, and $n_{\mathrm{C}}$ are set to $2.00 \times 10^{14}, 1.00$ $\times 10^{14}$, and $1.00 \times 10^{14} \mathrm{~cm}^{-3}$, respectively; $\phi_{1}$ and $\phi_{2}$ are set to $1.20 \mathrm{eV}$; proton conduction consists at the anode-electrolyte $\xi_{1}$ and cathodeelectrolyte $\xi_{2}$ interfaces are set to $1.27 \times 10^{-15} \mathrm{~A} / \mathrm{cm}^{2}$.

Overpotentials caused by the proton transfer from the anode to the cathode are evaluated using (22). In a practical system, these interfaces will have structures that are more complex and further incident events should be taken into account. However, as a first step, our model assumes simple interface structures and parameterizes the potential barriers at the interfaces. The energy dissipation in the bulk phases of electrodes and electrolyte membranes, $V_{\mathrm{R}}$, is assumed to be much smaller than that at the interfaces. That is, the overpotential caused by proton transfer is dominated by $V_{\mathrm{I}}$ so that the condition $V_{\mathrm{R}} \ll V_{\mathrm{I}}$ is satisfied. In addition, the present model does not take into account the effects of activation overpotential caused by chemical reactions on activating $\mathrm{H}_{2}$ and $\mathrm{O}_{2}$ gases and generating protons at the electrodes. Therefore, in accordance with the conventional procedure, the Butler-Volmer equation is used to evaluate the activation overpotential [12]:

$$
j=j_{0}\left[\exp \left[\frac{a q V_{\mathrm{BV}}}{k_{\mathrm{B}} T}\right]-\exp \left[-\frac{(1-a) q V_{\mathrm{BV}}}{k_{\mathrm{B}} T}\right]\right],
$$

where $a$ is the transfer coefficient. Using (23), $V_{\mathrm{BV}}$ can be numerically determined by the Newton-Raphson method. Therefore, in the present study, the total overpotential is simply given by

$$
V_{\text {tot }}=V_{\mathrm{I}}+V_{\mathrm{BV}}+V_{\mathrm{R}}
$$

\section{RESUlTS AND DISCUSSION}

Fig. 2 shows a schematic illustration of proton transfer together with the parameters used to compute the overpotential from (18)-(21). In particular, it shows the initial conditions and potential barrier heights at the electrode-electrolyte interfaces of a reference state. The parameters of this reference state are: $n_{\mathrm{A}}=2.00 \times 10^{14} \mathrm{~cm}^{-3}$, $n_{\mathrm{E}}=1.00 \times 10^{14} \mathrm{~cm}^{-3}, n_{\mathrm{C}}=1.00 \times 10^{14} \mathrm{~cm}^{-3}, \phi_{1}=1.20 \mathrm{eV}, \phi_{2}$ $=1.20 \mathrm{eV}, T=303 \mathrm{~K}, \xi_{1}=\xi_{2}=1.27 \times 10^{-15} \mathrm{~A} / \mathrm{cm}^{2}$, and $m=1$ (in mass numbers), where $n_{\mathrm{A}}, n_{\mathrm{E}}$, and $n_{\mathrm{C}}$ are given for equilibrium. Fig. 3 shows the overpotential computed using 


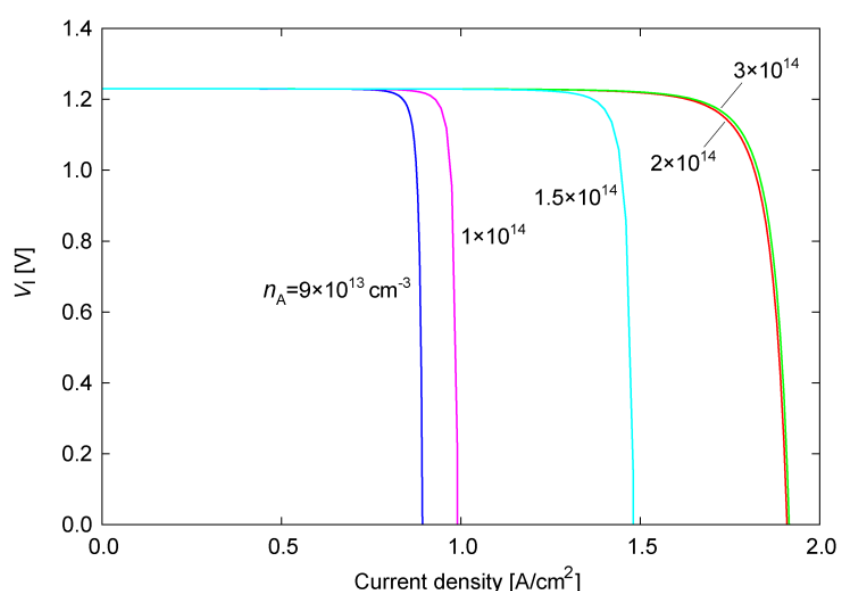

(a)

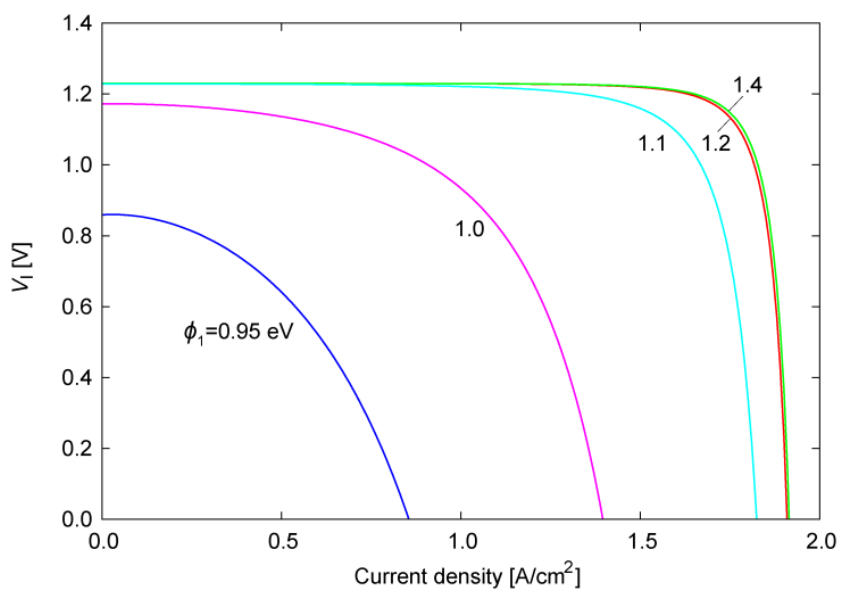

(c)

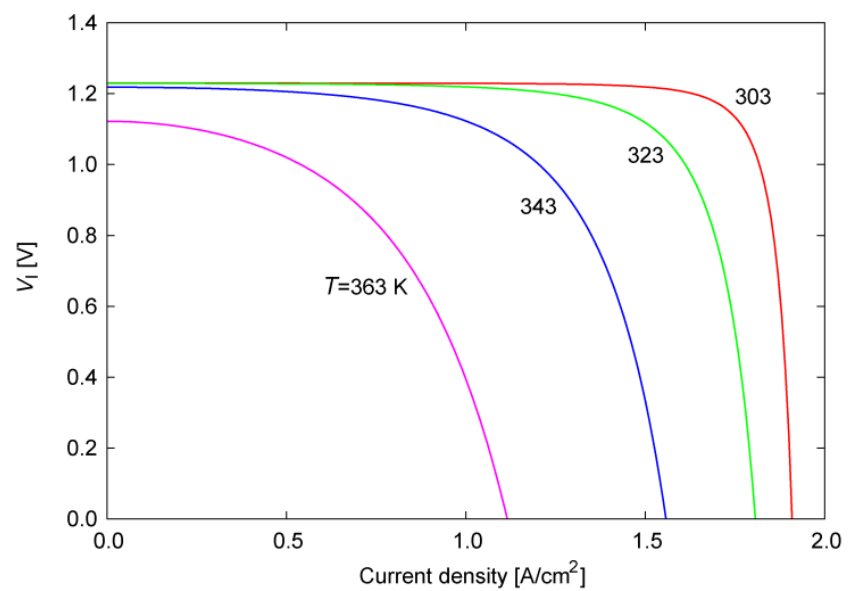

(e)

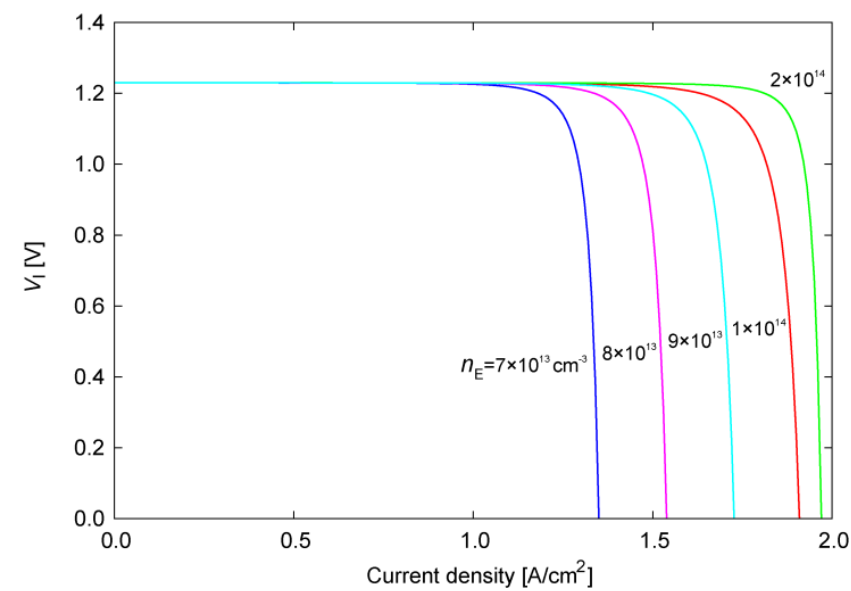

(b)

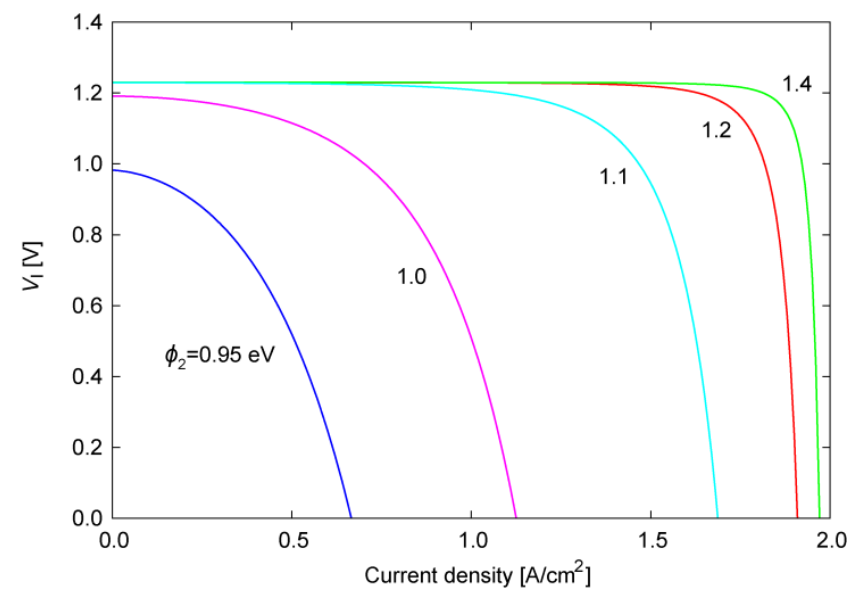

(d)

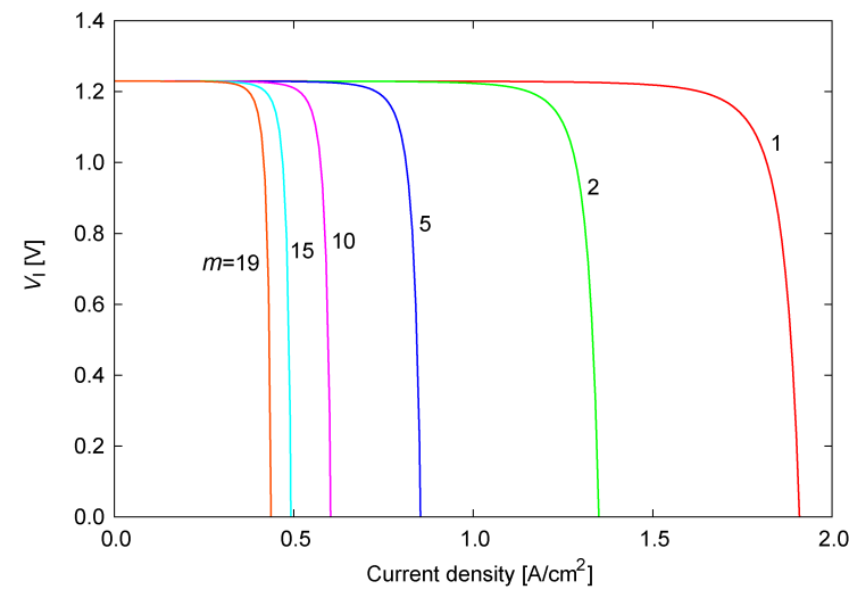

(f)

Fig. 3. Current-voltage characteristics resulting from (18)-(21) depending on (a) $n_{\mathrm{A}}$, (b) $n_{\mathrm{E}}$, (c) $\phi_{1}$, (d) $\phi_{2}$, (e) $T$, and (f) $m$.

the parameters. The voltage losses presented are relative to the ideal output voltage of a PEFC: 1.23 V. Fig. 3(a) shows the influence of $n_{\mathrm{A}}$ on the overpotential. Compared with the reference state of $2.00 \times 10^{4} \mathrm{~cm}^{-3}$, the limiting current density tends to decay as the proton concentration decreases. In particular, there appears to be a significant difference in the maximum current densities for number densities of $1.00 \times$ $10^{14}$ and $2.00 \times 10^{14} \mathrm{~cm}^{-3}$. When the concentration is lower than $2.00 \times 10^{14} \mathrm{~cm}^{-3}$, insufficient proton supply is predicted to cause serious degradation. In membrane electrode assemblies (MEAs), dilute protons are accelerated (thereby increasing the mean velocity) so as to maintain a constant current density. Consequently, the energy dissipation caused by inelastic collisions at the anode-electrolyte interface is critical. On the other hand, the current-voltage characteristics seem to converge at a proton concentration of $3.00 \times 10^{14}$ $\mathrm{cm}^{-3}$. Fig. 3(b) shows the effect of $n_{\mathrm{E}}$ on the overpotential. As shown in Fig. 3(a), the electrical characteristics improve as $n_{\mathrm{E}}$ increases. However, in this case, protons are supplied by the anode and they move to the cathode. Depending on the proton flow, the extreme decay observed at low proton concentrations can be avoided. Fig. 3(c) shows the effect of $\phi_{1}$ on the overpotential. The power generation efficiency decreases as $\phi_{1}$ decreases. This result seems to differ from the 


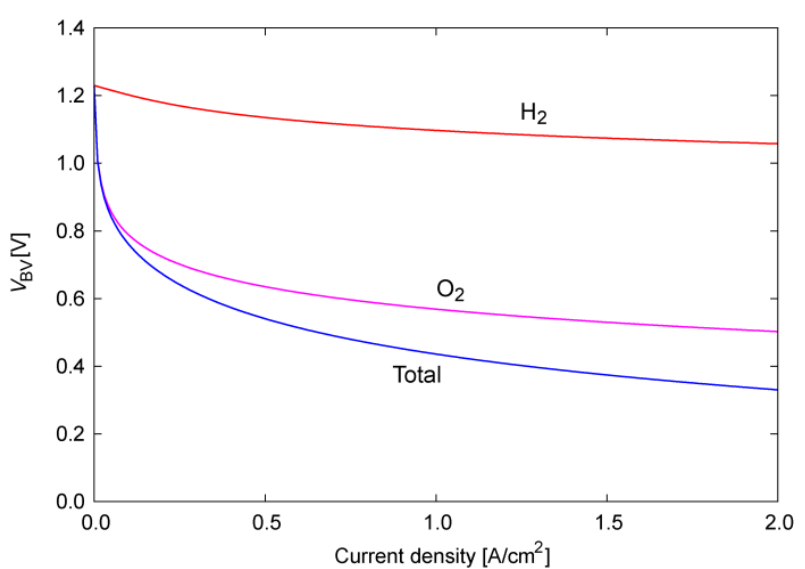

Fig. 4. Activation overpotential as a function of current density resulting from the Butler-Volmer equation (23). $a$ and $j_{0}$ are set to 0.50 and 0.10 $\mathrm{A} / \mathrm{cm}^{2}$ for $\mathrm{H}_{2}$ respectively and are 0.30 and $0.0010 \mathrm{~A} / \mathrm{cm}^{2}$ for $\mathrm{O}_{2}$, respectively.

behavior of real systems because potential barriers against conducting protons are the main cause of voltage losses. In this case, the proton concentrations in the anode and cathode are fixed at $2.00 \times 10^{14}$ and $1.00 \times 10^{14} \mathrm{~cm}^{-3}$ respectively. These quantities must be optimized for steady states. In addition, the interface conditions need to be determined more precisely by experimental and theoretical evaluations. However, the purpose of the present study is to develop a theoretical model that explains the overpotential caused by inelastic collisions of conducting protons in a PEFC. Therefore, the detailed interface conditions are left for future study. Fig. 3(d) shows the effect of $\phi_{2}$ on the overpotential. The current-voltage characteristics are similar to those for $\phi_{1}$. The overpotential increases as the potential barrier decreases. Fig. 3(e) shows the effect of $T$ on the overpotential. As the temperature increases, the output voltage decays remarkably due to the overpotential. This is because the energy dissipation of protons at the electrode-electrolyte interfaces generates a large voltage loss as the number of thermally excited protons increase. Accelerated protons collide inelastically with the interfaces and are decelerated, losing their kinetic energies. Eventually, they converge at stationary conditions in the adjacent layer, being frequently accelerated and decelerated in the interface regions. Fig. 3(f) shows the effect of $m$ on the overpotential. In the present model, the overpotential is also influenced by the carrier mass according to (18)-(21). The power supply efficiency decreases with increasing carrier mass. For example, there is a very large difference in the overpotential obtained for protons $(m=1)$ and oxonium ions $\mathrm{H}_{3} \mathrm{O}^{+}(m=19)$. This result means that the carrier conduction of oxonium ions (such as by the vehicle mechanism [13]) is expected to be inferior to that of protons. Increasing the carrier mass causes a large energy loss at the heterointerface. Fig. 4 shows the characteristics obtained using the Butler-Volmer equation (i.e., (23)). The activation

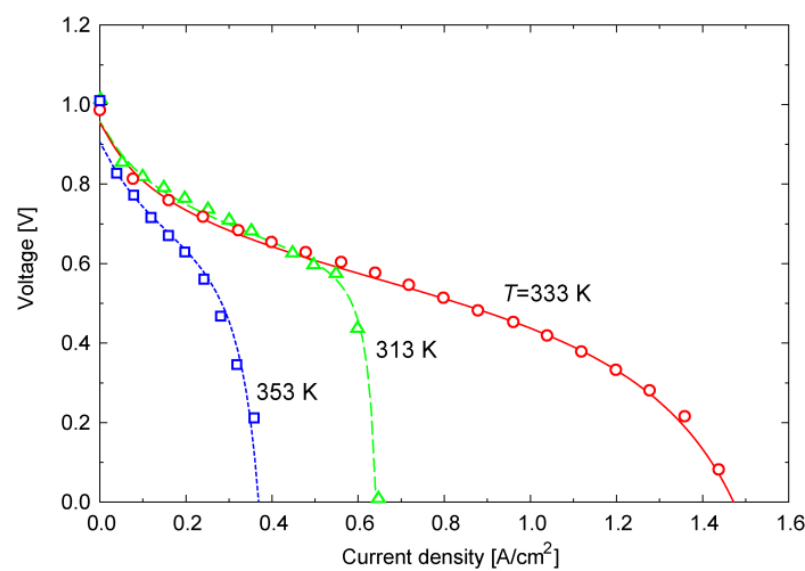

Fig. 5. Current-voltage characteristics measured at three different temperatures. Closed triangles, circles, and squares show the experimental results at 313,333 , and $353 \mathrm{~K}$, respectively. The lines present the theoretical results for the three experimental results.

overpotential for $\mathrm{O}_{2}$ molecules is larger than that for $\mathrm{H}_{2}$. Previous studies have estimated the binding energies of $\mathrm{O}_{2}$ and $\mathrm{H}_{2}$ to be $5.115 \mathrm{eV}(493.5 \mathrm{~kJ} / \mathrm{mol})$ and $4.478 \mathrm{eV}(432.1$ $\mathrm{kJ} / \mathrm{mol}$ ) [14], respectively. This difference in binding energies explains the difference in the activation energies for dissociating these molecules. In the present case, $a=0.50$ and $j_{0}=0.10 \mathrm{~A} / \mathrm{cm}^{2}$ for $\mathrm{H}_{2} ; a=0.30$ and $j_{0}=0.0010 \mathrm{~A} / \mathrm{cm}^{2}$ for $\mathrm{O}_{2}$. $T$ is set to $333 \mathrm{~K}$. As described above, the total of the overpotential in a PEFC is obtained by summing the parameters in (24).

This theoretical model is used to analyze experimental results in terms of atomistic-scale phenomena. Fig. 5 shows the current-voltage characteristics measured at several temperatures. The data points represent experimental results and the solid lines represent the theoretical results. In this experiment, the current-voltage characteristics were measured using an electrochemical impedance meter (KFM2150 and PLZ1004W; Kikusui Electronics, Inc.) for a Nafion ${ }^{\circledR} 212$ electrolyte membrane that was approximately $50 \mu \mathrm{m}$ thick. Each layer of the MEA was hot pressed and the electrode-electrolyte interfaces were tightly bound. The MEA was humidified at atmospheric pressure. The experimental results show that the output voltage decays as the temperature increases. The theoretical results can be fitted with the experimental results. Protons were assumed to be the carrier ions in the MEA so the carrier mass was set to $m=1$ (in mass numbers). The results for $333 \mathrm{~K}$ show that the voltage decreases steeply as the current density increases to near $0.3 \mathrm{~A} / \mathrm{cm}^{2}$. This is caused by the activation overpotentials for $\mathrm{H}_{2}$ and $\mathrm{O}_{2}$. The voltage loss is linear between 0.3 and $1.0 \mathrm{~A} / \mathrm{cm}^{2}$. In this region, the currentvoltage characteristics are considered to be ohmic. The output voltage decreases rapidly at current densities over 1.0 $\mathrm{A} / \mathrm{cm}^{2}$. This reduction is due to insufficient supply of protons in each layer. In the present model, this reduction is caused by

TABLE I: Parameters Used to ANALyze the ExPerimental Results in Fig. 5

\begin{tabular}{|c|c|c|c|c|c|c|c|c|c|c|c|}
\hline \multirow[b]{2}{*}{$T[\mathrm{~K}]$} & \multirow[b]{2}{*}{$\phi_{1}[\mathrm{eV}]$} & \multirow[b]{2}{*}{$\phi_{2}[\mathrm{eV}]$} & \multirow[b]{2}{*}{$n_{\mathrm{A}}\left[\mathrm{cm}^{-3}\right]$} & \multirow[b]{2}{*}{$n_{\mathrm{E}}\left[\mathrm{cm}^{-3}\right]$} & \multirow[b]{2}{*}{$n_{\mathrm{C}}\left[\mathrm{cm}^{-3}\right]$} & \multirow[b]{2}{*}{$\xi_{1}\left[\mathrm{~A} / \mathrm{cm}^{2}\right]$} & \multirow[b]{2}{*}{$\xi_{2}\left[\mathrm{~A} / \mathrm{cm}^{2}\right]$} & \multicolumn{2}{|c|}{$\mathrm{H}_{2}$} & \multicolumn{2}{|c|}{$\mathrm{O}_{2}$} \\
\hline & & & & & & & & $j_{0}\left[\mathrm{~A} / \mathrm{cm}^{2}\right]$ & $a$ & $j_{0}\left[\mathrm{~A} / \mathrm{cm}^{2}\right]$ & $a$ \\
\hline 313 & 0.508 & 0.484 & $6.50 \times 10^{13}$ & $5.50 \times 10^{13}$ & $1.00 \times 10^{13}$ & $1.29 \times 10^{-6}$ & $1.29 \times 10^{-6}$ & 0.100 & 0.50 & 0.020 & 0.40 \\
\hline 333 & 0.508 & 0.484 & $1.65 \times 10^{14}$ & $1.12 \times 10^{14}$ & $5.00 \times 10^{13}$ & $2.27 \times 10^{-6}$ & $2.27 \times 10^{-6}$ & 0.100 & 0.50 & 0.020 & 0.40 \\
\hline 353 & 0.508 & 0.484 & $6.25 \times 10^{14}$ & $2.05 \times 10^{13}$ & $1.00 \times 10^{13}$ & $2.20 \times 10^{-6}$ & $2.20 \times 10^{-6}$ & 0.100 & 0.50 & 0.020 & 0.40 \\
\hline
\end{tabular}


TABLE II: PARAMETERS USED TO ANALYZE THE EXPERIMENTAL RESULTS IN FIG. 6

\begin{tabular}{|c|c|c|c|c|c|c|c|c|c|c|c|}
\hline & \multirow[b]{2}{*}{$\phi_{1}[\mathrm{eV}]$} & \multirow[b]{2}{*}{$\phi_{2}[\mathrm{eV}]$} & \multirow[b]{2}{*}{$n_{\mathrm{A}}\left[\mathrm{cm}^{-3}\right]$} & \multirow[b]{2}{*}{$n_{\mathrm{E}}\left[\mathrm{cm}^{-3}\right]$} & \multirow[b]{2}{*}{$n_{\mathrm{C}}\left[\mathrm{cm}^{-3}\right]$} & \multirow[b]{2}{*}{$\xi_{1}\left[\mathrm{~A} / \mathrm{cm}^{2}\right]$} & \multirow[b]{2}{*}{$\xi_{2}\left[\mathrm{~A} / \mathrm{cm}^{2}\right]$} & \multicolumn{2}{|c|}{$\mathrm{H}_{2}$} & \multicolumn{2}{|c|}{$\mathrm{O}_{2}$} \\
\hline & & & & & & & & $j_{0}\left[\mathrm{~A} / \mathrm{cm}^{2}\right]$ & $a$ & $j_{0}\left[\mathrm{~A} / \mathrm{cm}^{2}\right]$ & $a$ \\
\hline Hot-pressed & 0.508 & 0.484 & $1.65 \times 10^{14}$ & $1.12 \times 10^{14}$ & $5.00 \times 10^{13}$ & $2.27 \times 10^{-6}$ & $2.27 \times 10^{-6}$ & 0.100 & 0.50 & 0.020 & 0.40 \\
\hline Non-pressed & 0.615 & 0.594 & $1.70 \times 10^{15}$ & $4.88 \times 10^{13}$ & $1.00 \times 10^{13}$ & $8.00 \times 10^{-8}$ & $8.00 \times 10^{-8}$ & 0.080 & 0.50 & 0.010 & 0.40 \\
\hline
\end{tabular}

protons passing through the potential barriers with high kinetic energies and then dissipating a lot of their energy due to collisions with the electrode-electrolyte interfaces. Table I shows the values of the parameters used in the theoretical model, where the temperatures $(313,333$, and $353 \mathrm{~K})$ are the experimental temperatures. In this computation, the potential barriers of $\phi_{1}$ and $\phi_{2}$ were set to 0.508 and $0.484 \mathrm{eV}$, respectively. To analyze the experimental results, the potential barrier heights, which depend on the interface structures, are parameterized; however, these quantities should be determined by first-principles computations. The experimental results reveal that output at $333 \mathrm{~K}$ is higher than those at 313 and $353 \mathrm{~K}$. This high output is attributed to there being sufficient protons in each layer. That is, an appropriate distribution of proton concentrations enhances the performance. In this case, $n_{\mathrm{A}}, n_{\mathrm{E}}$, and $n_{\mathrm{C}}$ are determined as $1.65 \times 10^{14}, 1.12 \times 10^{14}$, and $5.00 \times 10^{14} \mathrm{~cm}^{-3}$, respectively. In addition, the current densities $\xi_{1}$ and $\xi_{2}$ that contribute to the voltage losses are estimated to be $2.27 \times 10^{-6} \mathrm{~A} / \mathrm{cm}^{2}$ in each layer. On the other hand, for $313 \mathrm{~K}, n_{\mathrm{A}}, n_{\mathrm{E}}$, and $n_{\mathrm{C}}$ are evaluated to be $6.50 \times 10^{13}, 5.50 \times 10^{13}$, and $1.00 \times 10^{13} \mathrm{~cm}^{-3}$, respectively. These values are less than those at $333 \mathrm{~K}$. Insufficient proton supply greatly increases the voltage loss, as shown in Figs. 3(a) and (b). In addition, proton conduction across the electrode-electrolyte interfaces, $\xi_{1}=\xi_{2}=1.29 \times$ $10^{-6} \mathrm{~A} / \mathrm{cm}^{2}$, is predicted to be lower than at the other two temperatures. At low temperatures, insufficient proton supply and inactivation of proton conduction at the interfaces reduces the output voltage. In the result for $353 \mathrm{~K}$, the output voltage falls off rapidly at a low current density. In this case, there is a remarkable difference between the proton concentrations in the anode and the electrolyte $\left(6.25 \times 10^{14}\right.$ $\mathrm{cm}^{-3}$ and $2.05 \times 10^{13} \mathrm{~cm}^{-3}$, respectively). The high proton concentration increases the amount of high-energy protons. Furthermore, a large velocity difference gives rise to great energy dissipation at the interface, according to (16) and (17). In addition, the velocity distribution becomes broader at higher temperatures. As a result, increasing the temperature increases the amount of high-energy protons.

Fig. 6 shows the results of current-voltage and currentpower density measurements. The experimental results are represented by data points and the theoretical calculations are indicated by solid lines. The characteristics of two MEAs with different electrode-electrolyte interfaces are compared. One of them is the measurement results at $333 \mathrm{~K}$ shown in Fig. 5. In this MEA, the electrodes and the electrolyte membrane are hot pressed and the interfaces are physically adsorbed. The other MEA has simple interfaces in which the anode, electrolyte membrane, and the cathode are stacked without being hot pressed. The amount of $\mathrm{Pt}$ added as a catalyst and the quality of electrolyte membrane of Nafion ${ }^{\circledR}$ 212 are the same as those in the hot-pressed MEA. In both cases, the temperature was $333 \mathrm{~K}$. These two MEAs were used to investigate the effects of the interfaces on the overvoltage. Fig. 6 shows that the MEA with non-hot-pressed interfaces has poorer properties than that with hot-pressed ones. The maximum power densities obtained using the hot-pressed and non-hot-pressed MEAs are $0.435 \mathrm{~W} / \mathrm{cm}^{2}$ at $0.960 \mathrm{~A} / \mathrm{cm}^{2}$ and $0.243 \mathrm{~W} / \mathrm{cm}^{2}$ at $0.558 \mathrm{~A} / \mathrm{cm}^{2}$, respectively. In particular, in the case of the non-hot-pressed MEA, a high activation overpotential is observed at low current densities and the output voltage decays drastically at slightly higher current densities. These differences are definitely related to the condition of the interfaces. Table II lists the parameters used in the theoretical computations. The potential barriers at the interfaces of the non-hot-pressed MEA are approximately $0.1 \mathrm{eV}$ higher than those of the hot-pressed one. This result demonstrates that poor interface conditions disrupt the proton transport. The proton concentrations in each layer are non-optimal due to degradation of the interface conditions. Consequently, the large differences in the proton concentration induce a significant overpotential. In particular, the proton concentration in the anode $\left(1.70 \times 10^{15} \mathrm{~cm}^{-3}\right)$ is greater than that in the electrolyte $\left(4.88 \times 10^{13} \mathrm{~cm}^{-3}\right)$. The interface degradation also reduces the parameters $\xi_{1}$ and $\xi_{2}$, which are evaluated to be $8.00 \times 10^{-8} \mathrm{~A} / \mathrm{cm}^{2}$. These quantities are much less than those for high-quality interfaces. In addition, the reduction in the current density in the ButlerVolmer equation (i.e., (23)) is taken into account. As mentioned above, degradation of the interfaces greatly affects the current-voltage characteristics. Using the two MEAs with different interfaces it was possible to clarify the relationship between the overpotential and the electrodeelectrolyte interface conditions.

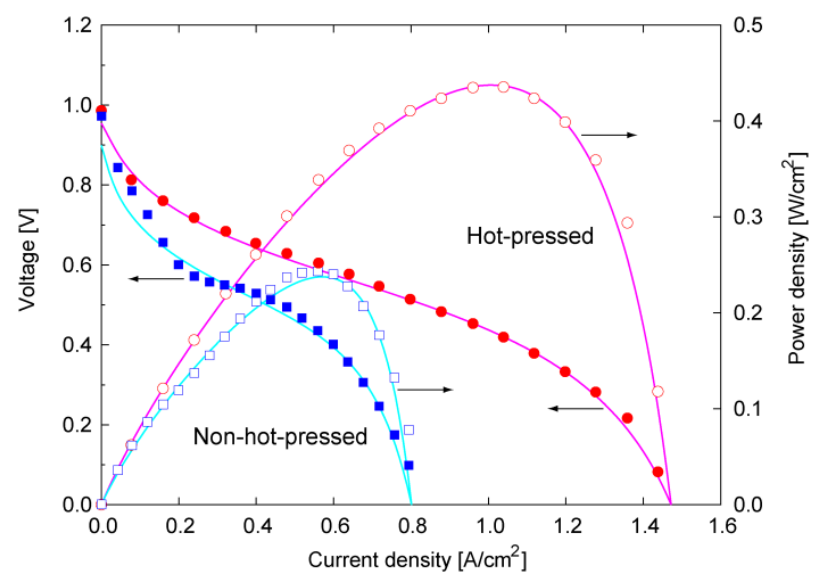

Fig. 6. Comparison of current-voltage characteristics between the hotpressed and non-hot-pressed MEAs. Data points and solid lines show the experimental results and theoretical analysis, respectively. Closed circles and closed squares indicate the output voltage as a function of current density with and without interface treatments of MEAs; open circles and open squares show the power density with and without interface treatments, respectively.

In a previous study [15], we supposed that collisions of charge carriers at surfaces namely cause the energy losses. The present results also confirmed the overpotential due to 
inelastic collisions at interfaces. Our studies have contributed to theoretically reveal energy dissipations in nonequilibrium conditions. In our future work, it is intended to combine the present model with first-principles methods [16], [17] and molecular dynamics simulations [18]-[20]. Such challenging topics will permit the interface conditions to be investigated quantitatively in more detail, following the dynamics of proton conduction.

\section{CONCLUSION}

In the present study, we developed a novel theoretical model for an overpotential due to proton transport in PEFCs. This model predicted that energy losses in PEFCs were caused by inelastic collisions of protons at the electrodeelectrolyte interfaces. The proton kinetics were treated in terms of atomistic-scale phenomena. Effects of several model parameters on the overpotential were investigated. In addition, the current-voltage characteristics were measured for two different MEAs. The evaluations were in good agreement with the experimental results and they clearly revealed that the interface conditions affected the performance of PEFCs. In particular, the results suggest it is important to optimize the proton concentrations in the electrodes and the electrolyte membrane to realize good performance. Poor interface conditions increased the potential barriers for conducting protons and disrupted the favorable distribution of protons. Excess concentration and depletion of protons seriously degraded the output voltage, mainly due to the overpotential. Consequently, we suppose that electrode-electrolyte interfaces are a dominant factor in determining the overpotential in PEFCs as well as activation overpotentials. Furthermore, the results imply that it is essential to improve the interface conditions to obtain good proton conduction.

\section{REFERENCES}

[1] A. B. Bose, R. Shaik, and J. Mawdsley, "Optimization of the performance of polymer electrolyte fuel cell membrane-electrode assemblies: Roles of curing parameters on the catalyst and ionomer structures and morphology," J. Power Sources, vol. 182, pp. 61-65, 2008.

[2] N. Wagner, T. Kaz, and K. A. Friedrich, "Investigation of electrode composition of polymer fuel cells by electrochemical impedance spectroscopy," Electrochim. Acta, vol. 53, pp. 7475-7482, 2008.

[3] M. W. Verbrugge and R. F. Hill, "Ion and solvent transport in ion-exchange membranes, I. a macrohomogeneous mathematical model," J. Electrochem. Soc., vol. 137, pp. 886-893, 1990.
[4] M. W. Verbrugge and R. F. Hill, "Ion and solvent transport in ion-exchange membranes, II. a radiotracer study of the sulfuric-acid, nafion-117 system," J. Electrochem. Soc., vol. 137, pp. 893-899, 1990

[5] C.-Y. Wang, "Fundamental models for fuel cell engineering," Chem. Rev. vol. 104, pp. 4727-4766, 2004.

[6] J. Ramousse, J. Deseure, O. Lottin, S. Didierjean, and D. Maillet, "Modelling of heat, mass and charge transfer in a PEMFC single cell," J. Power Sources, vol. 145, pp. 416-427, 2005.

[7] Z. Zhang, X. Wang, X. Zhang, and L. Jia, "Optimizing the performance of a single pem fuel cell," J. Fuel Cell Sci. Technol., vol. 5, p. 031007 , 2008 .

[8] D. Marx, M. E. Tuckerman, J. Hutter, and M. Parrinello, "The nature of the hydrated excess proton inwater," Nature, vol. 397, pp. 601-604, 1999.

[9] N. Agmon, "Mechanism of hydroxide mobility," Chem. Phys. Lett. vol. 319, pp. 247-252, 2000.

[10] M. E. Tuckerman, D. Marx, and M. Parrinello, "The nature and transport mechanism of hydrated hydroxide ions in aqueous solution," Nature, vol. 417, pp. 925-929, 2002.

[11] J. A. Elliott and S. J. Paddison, "Modelling of morphology and proton transport in PFSA membranes," Phys. Chem. Chem. Phys., vol. 9, pp. 2602-2618, 2007.

[12] R. Óhayre, S.-W. Cha, W. Colella, and F. B. Prinz, Fuel Cell Fundamentals, 2nd ed. New York: John Wiley \& Sons, 2009, pp. 8388.

[13] K. D. Kreuer, "On the development of proton conducting materials for technological applications,” Solid State Ionics, vol. 97, pp. 1-15, 1997.

[14] H. Okabe, Photochemistry of Small Molecules, New York: John Wiley \& Sons, 1978, pp. 162\&177.

[15] K. Doi, Y. Nishioka, and S. Kawano, "Theoretical study of electric current in DNA base molecules trapped between nanogap electrodes," Comput. Theor. Chem., vol. 999, pp. 203-214, 2012.

[16] K. Doi et al., "Ab initio calculation of electron effective masses in solid pentacene," J. Appl. Phys., vol. 98, p. 113709, 2005.

[17] K. Doi, T. Yonebayashi, and S. Kawano, "Perturbation theory analysis for electronic response of DNA base pairs," J. Mol. Struct.: THEOCHEM, vol. 939, pp. 97-105, 2010.

[18] H. Nakano, H. Ohta, A. Yokoe, K. Doi, and A. Tachibana, "First-principle molecular-dynamics study of hydrogen adsorption on an aluminum-doped carbon nanotube," J. Power Sources, vol. 163, pp. 125-134, 2006.

[19] K. Doi, H. Nakano, H. Ohta, and A. Tachibana, "First-principle molecular-dynamics study of hydrogen and aluminum nanowires in carbon nanotubes," Mater. Sci. Forum, vol. 539-543, pp. 1409-1414, 2007.

[20] K. Doi, I. Onishi, and S. Kawano, "Dissociative adsorption of $\mathrm{H}_{2}$ molecules on steric graphene surface: Ab initio MD study based on DFT," Comput. Theor. Chem., vol. 994, pp. 54-64, 2011.

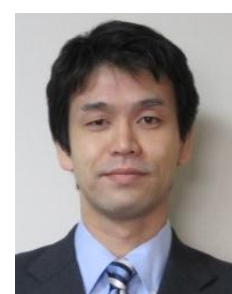

Kentaro Doi received his $\mathrm{PhD}$ in mechanical engineering from Kyoto University in 2007. He studied $a b$ initio methods to treat electronic properties of molecules and solids associated with semiconductor devices. He is presently an associate professor in the Department of Mechanical Science and Bioengineering, Osaka University. His current research interests focus on dynamics of molecules interacting with liquids in micro/nanofluidic devices. 